Georgian Mathematical Journal

Volume 14 (2007), Number 1, 81-97

\title{
COMPLETENESS THEOREMS FOR ELLIPTIC EQUATIONS OF HIGHER ORDER WITH CONSTANT COEFFICIENTS
}

\author{
ALBERTO CIALDEA
}

To the Memory of Prof. Gaetano Fichera

\begin{abstract}
Let $\left\{\omega_{k}\right\}$ be a complete system of polynomial solutions of the elliptic equation $\sum_{|\alpha| \leqslant 2 m} a_{\alpha} D^{\alpha} u=0, a_{\alpha}$ being real constants. We give necessary and sufficient conditions for the completeness of the system $\left\{\left(\omega_{k}, \partial_{\nu} \omega_{k}\right.\right.$, $\left.\left.\ldots, \partial_{\nu}^{m-1} \omega_{k}\right)\right\}$ in $\left[L^{p}(\partial \Omega)\right]^{m}$, where $\Omega \subset \mathbb{R}^{n}$ is a bounded domain such that $\mathbb{R}^{n} \backslash \bar{\Omega}$ is connected and $\partial \Omega \in C^{1}$.
\end{abstract}

2000 Mathematics Subject Classification: 42C30, 35J30, 31B10.

Key words and phrases: Completeness theorems, higher order elliptic equations, potential theory.

\section{INTRODUCTION}

Many years ago Mauro Picone posed the following problem: let $E$ be a partial differential operator

$$
E u=\sum_{|\alpha| \leqslant 2 m} a_{\alpha}(x) D^{\alpha} u
$$

defined in $\mathbb{R}^{n}$ and let $B_{1}, \ldots, B_{s}$ be some partial differential operators defined on the boundary $\Sigma$ of a bounded domain $\Omega$. Let us suppose that there exists a solution of the problem

$$
\begin{cases}E u=0 & \text { in } \Omega \\ B_{h} u=f_{h} & \text { on } \Sigma(h=1, \ldots, s)\end{cases}
$$

if and only if $\left(f_{1}, \ldots, f_{s}\right)$ satisfies a finite number of compatibility conditions

$$
\sum_{h=1}^{s} \int_{\Sigma} f_{h} \psi_{h}^{(k)} d \sigma=0, \quad k=1, \ldots, \mu,
$$

that is to say that problem (1.1) is an index problem.

Let us denote by $\left\{\omega_{k}\right\}$ a particular sequence of solutions of the equation $E u=0$ in $A$, where $A$ is a domain such that $\bar{\Omega} \subset A$.

The problem posed by Picone is to find under which conditions the system $\left\{\left(B_{1} \omega_{k}, \ldots, B_{s} \omega_{k}\right)\right\}$ is complete in the space

$$
\left\{\left(v_{1}, \ldots, v_{s}\right) \in\left[L^{p}(\Sigma)\right]^{s} \mid \sum_{h=1}^{s} \int_{\Sigma} v_{h} \psi_{h}^{(k)} d \sigma=0, k=1, \ldots, \mu\right\} .
$$


The first theorem of such a kind was proved by Gaetano Fichera in [7]. He considered the Dirichlet, the Neumann and the mixed problem for the Laplace equation in any number of variables and proved the corresponding completeness of the harmonic polynomials in $L^{p}(\Sigma)$.

The history of the problem posed by Picone and the connections with more classical approximation problems of Mergelyan and Runge type can be found in [10]. That paper contains a complete list of references. See also [5] for an update.

No general completeness theorems in the sense of Picone have so far been known. There are several results available only for particular partial differential equations and for particular boundary value problems, except for elliptic equations of second order and for some results in two independent variables. The present paper and [4] seem to be the first ones to consider general classes of partial differential operators of higher order in any number of variables.

We consider an elliptic equation of higher order with constant real coefficients

$$
E u=0,
$$

where

$$
E u=\sum_{|\alpha| \leqslant 2 m} a_{\alpha} D^{\alpha} u \quad\left(a_{\alpha} \in \mathbb{R}\right) .
$$

The theory of partial differential equations with constant coefficients attracts a great deal of attention (for general references, see [23]). Here we prove the completeness theorems for the Dirichlet problem

$$
\begin{cases}E u & \text { in } \Omega, \\ \partial_{\nu}^{h} u=f_{h} & \text { on } \Sigma, h=0, \ldots, m-1\end{cases}
$$

where $\Omega$ is a bounded domain of $\mathbb{R}^{n}$ such that $\mathbb{R}^{n} \backslash \bar{\Omega}$ is connected, $\Sigma$ is its boundary and $\partial_{\nu}$ denotes the normal derivative. It is worthwhile to remark that $\Sigma$ is merely supposed to be a $C^{1}$ boundary.

It is easy to see that there are polynomial solutions of the equation $E u=0$ if and only if $a_{(0 \ldots 0)}=0$ in (1.3). But, generally speaking, this condition is not sufficient for the completeness. The main result we prove is the following:

Let $a_{(0 \ldots 0)}=0$ and let $E$ be such that the Garding inequality holds (see (3.2) below). Let us denote by $\left\{\omega_{k}\right\}$ a complete system of polynomial solutions of (1.2). The system

$$
\left\{\left(\omega_{k}, \partial_{\nu} \omega_{k}, \ldots, \partial_{\nu}^{m-1} \omega_{k}\right)\right\}
$$

is complete in the space $\left[L^{p}(\Sigma)\right]^{m}(1 \leqslant p<\infty)$ if and only if all the irreducible factors $($ over $\mathbb{C}$ ) of the characteristic polynomial vanish at $\xi=0$.

The particular case of an elliptic operator with only highest order terms was considered in [4].

Our proof is based, on the one hand, on the fundamental results obtained by Malgrange in [16] and, on the other hand, on some formulas of potential theory we give in Section 2 . 
Another ingredient of the proof is the construction of a suitable fundamental solution for operator (1.3). In the case of an elliptic operator with constant coefficients and no lower order terms, such a fundamental solution is provided by the one constructed by Fritz John in [13] (see [4]).

In the general case it is well known that Ehrenpreis [6] and Malgrange [16] proved that there exists a fundamental solution for any partial differential operator with constant coefficients (see [17] for a short proof). Afterwarda, several explicit representations have been obtained (see Chapter 3 of [23] and [11, 13, $14,18,24])$.

Unfortunately none of these representations seems to be suitable for our purposes. This is why we are going to construct a different fundamental solution, which we shall call a principal fundamental solution in the sense of Fichera. The idea, which simplifies the classical concept of a principal fundamental solution given by Giraud, was introduced in [8] with the aim of developing a multiple layer potential theory for elliptic differential equations of higher order with variable coefficients in two variables. Later this construction was generalized to strongly elliptic systems with variable coefficients in any number of variables [20].

The construction of this fundamental solution is carried out in Section 3. We could not apply directly the results of $[8,20]$, because we cannot impose the conditions they require on the term $a_{(0, \ldots, 0)}$. On the other hand, the fact that we have constant coefficients will permit us to adapt and simplify Fichera's construction.

\section{Some Results of Potential Theory}

We recall that the function $h$ is said to be essentially homogeneous of degree $\alpha$ if $h(x)=h_{1}(x) \log x+h_{2}(x)$ where $h_{2}(\varrho x)=\varrho^{\alpha} h(x), x \neq 0, \varrho>0$ and $h_{1}(x)$ is a homogeneous polynomial of degree $\alpha$ if $\alpha$ is a nonnegative integer, $h_{1}(x) \equiv 0$ otherwise.

In [3] the following result was proved.

Theorem 1. Let $\Sigma \in C^{1, \lambda}$. Let $h \in C^{2}\left(\mathbb{R}^{n} \backslash\{0\}\right)$ be even and essentially homogeneous of degree $2-n$. If $\varphi \in L^{1}(\Sigma)$ and $x_{0}$ is a Lebesgue point for $\varphi$, then

$$
\begin{aligned}
\lim _{x \rightarrow x_{0}} \int_{\Sigma} \varphi(y) \partial_{x_{k}}[h(x-y)] d \sigma_{y}= & \nu_{k}\left(x_{0}\right) \gamma\left(x_{0}\right) \varphi\left(x_{0}\right) \\
& +\int_{\Sigma} \varphi(y) \partial_{x_{k}}[h(x-y)] d \sigma_{y}
\end{aligned}
$$

where $x$ is a point on the inner normal $\nu_{x_{0}}$ to $\Sigma$ at $x_{0}, x^{\prime}$ is its symmetric point with respect to $x_{0}$ and the last integral has to be understood as a singular 
integral. The function $\gamma$ is given by

$$
\gamma\left(x_{0}\right)= \begin{cases}\pi h_{1}-\frac{1}{2} \int_{|\xi|=1} \Delta h_{2}(\xi) \log \left|\xi \cdot \nu_{x_{0}}\right| d \sigma_{\xi} & \text { if } n=2 \\ \frac{1}{2} \int_{|\xi|=1}\left[(2-n) h(\xi)-\Delta h(\xi) \log \left|\xi \cdot \nu_{x_{0}}\right|\right] d \sigma_{\xi} & \text { if } n \geqslant 3 .\end{cases}
$$

Jump formulas like (2.1) are known, even for more general kernels (see [15, pp. 293-300]). The interest of Theorem 1 is in the explicit expression for $\gamma$; in fact, as it was remarked in [5], the function $\gamma$ can be expressed by means of the Fourier transform of the kernel $h$ :

$$
\gamma\left(x_{0}\right)=\frac{1}{2} \mathscr{F}(\Delta h)\left(\nu_{x_{0}}\right)=-2 \pi^{2} \mathscr{F}(h)\left(\nu_{x_{0}}\right)
$$

where the Laplacian $\Delta$ has to be understood in the sense of distributions and $\mathscr{F}$ denotes the Fourier transform

$$
\mathscr{F}(h)(x)=\int_{\mathbb{R}^{n}} h(y) e^{-2 \pi i x \cdot y} d y .
$$

By means of this formula it is often easy to find explicit formulas. For example, if we denote by $s$ the fundamental solution of the biharmonic equation

$$
s(x-y) \begin{cases}=\left[2 c_{n}(n-2)(n-4)\right]^{-1}|x-y|^{4-n} & \text { if } n=3,5,6, \ldots, \\ -\left(4 c_{4}\right)^{-1} \log |x| & \text { if } n=4\end{cases}
$$

from (2.1) we have

$$
\begin{aligned}
\lim _{x \rightarrow x_{0}} \int_{\Sigma} \varphi(y) \frac{\partial^{3}}{\partial x_{h} \partial x_{k} \partial x_{j}} s(x-y) d \sigma_{y} & \\
& =\nu_{h}\left(x_{0}\right) \gamma_{k j}\left(x_{0}\right) \varphi\left(x_{0}\right)+\int_{\Sigma} \varphi(y) \frac{\partial^{3}}{\partial x_{h} \partial x_{k} \partial x_{j}} s\left(x_{0}-y\right) d \sigma_{y},
\end{aligned}
$$

where

$$
\gamma_{k j}\left(x_{0}\right)=-2 \pi^{2} \mathscr{F}\left(\frac{\partial^{2} s}{\partial x_{k} \partial x_{j}}\right)\left(\nu_{x_{0}}\right) .
$$

On the other hand, since $\Delta^{2} s=\delta$, we have $16 \pi^{4}|x|^{4} \mathscr{F}(s)(x)=1$. This leads to

and we have

$$
\gamma_{k j}(x)=\frac{x_{k} x_{j}}{2|x|^{4}}
$$

$$
\begin{aligned}
\lim _{x \rightarrow x_{0}} \int_{\Sigma} \varphi(y) & \frac{\partial^{3}}{\partial x_{h} \partial x_{k} \partial x_{j}} s(x-y) d \sigma_{y} \\
= & \frac{1}{2} \nu_{h}\left(x_{0}\right) \nu_{k}\left(x_{0}\right) \nu_{j}\left(x_{0}\right) \varphi\left(x_{0}\right)+\int_{\Sigma} \varphi(y) \frac{\partial^{3}}{\partial x_{h} \partial x_{k} \partial x_{j}} s\left(x_{0}-y\right) d \sigma_{y} .
\end{aligned}
$$


This formula was proved in $[2$, Lemma $\mathrm{V}]$ in a direct, but more complicated way.

As another example, we can consider the double layer potential for the elasticity system $\Delta u+k \operatorname{grad} \operatorname{div} u$. If we denote by $s_{h j}$ the Somigliana matrix

$$
s_{h j}(x-y)=-\frac{1}{4 \pi}\left[\frac{\delta_{h j}}{|x-y|}-\frac{k}{2(1+k)} \frac{\partial^{2}}{\partial x_{j} \partial x_{h}}|x-y|\right],
$$

then the elastic double layer potential is given by

$$
u_{h}(x)=\int_{\Sigma} \varphi_{j}(y) L_{j y}\left[s^{h}(x-y)\right] d \sigma_{y},
$$

where $L_{j}$ is the operator

$$
L u=(k-1)(\operatorname{div} u) \nu+2 \frac{\partial u}{\partial \nu}+\nu \wedge \operatorname{rot} u
$$

and $s^{h}$ is the vector whose components are $s_{h j}$.

Formula (2.1) leads to

$$
\begin{aligned}
& \lim _{x \rightarrow x_{0}} \int_{\Sigma} \varphi_{j}(y) L_{j y}\left[s^{h}(x-y)\right] d \sigma_{y} \\
& \quad=-\left[k \nu_{i}\left(x_{0}\right) \nu_{j}\left(x_{0}\right)+\delta_{i j}\right] \gamma_{h i}\left(x_{0}\right) \varphi_{j}\left(x_{0}\right)+\int_{\Sigma} \varphi_{j}(y) L_{j y}\left[s^{h}\left(x_{0}-y\right)\right] d \sigma_{y}
\end{aligned}
$$

where

$$
\gamma_{h i}\left(x_{0}\right)=-2 \pi^{2} \mathscr{F}\left(s_{h i}\right)\left(\nu_{x_{0}}\right) .
$$

But since $s_{h i}$ is a fundamental solution of the elasticity system, we have $\left(-4 \pi^{2}\right)\left(|x|^{2} \delta_{i j}+k x_{i} x_{j}\right) \mathscr{F}\left(s_{h i}\right)(x)=\delta_{j h}$ and

$-\left[k \nu_{i}\left(x_{0}\right) \nu_{j}\left(x_{0}\right)+\delta_{i j}\right] \gamma_{h i}\left(x_{0}\right)=2 \pi^{2}\left[k \nu_{i}\left(x_{0}\right) \nu_{j}\left(x_{0}\right)+\delta_{i j}\right] \mathscr{F}\left(s_{h i}\right)\left(\nu_{x_{0}}\right)=-\frac{1}{2} \delta_{j h}$.

We have thus reobtained in a simple way the very well known formula

$$
\lim _{x \rightarrow x_{0}} \int_{\Sigma} \varphi_{j}(y) L_{j y}\left[s^{h}(x-y)\right] d \sigma_{y}=-\frac{1}{2} \varphi_{h}\left(x_{0}\right)+\int_{\Sigma} \varphi_{j}(y) L_{j y}\left[s^{h}\left(x_{0}-y\right)\right] d \sigma_{y} \text {. }
$$

The following theorem was proved in [5].

Theorem 2. Let $\Sigma \in C^{1}$. Let $h \in C^{2}\left(\mathbb{R}^{n} \backslash\{0\}\right)$ be even and essentially homogeneous of degree $2-n$. If $\varphi \in L^{1}(\Sigma)$ and $x_{0}$ is a Lebesgue point for $\varphi$, then

$$
\begin{gathered}
\lim _{x \rightarrow x_{0}}\left(\int_{\Sigma} \varphi(y) \partial_{x_{k}}[\right. \\
\left.h(x-y)] d \sigma_{y}-\int_{\Sigma} \varphi(y) \partial_{x_{k}}\left[h\left(x^{\prime}-y\right)\right] d \sigma_{y}\right) \\
=2 \nu_{k}\left(x_{0}\right) \gamma\left(x_{0}\right) \varphi\left(x_{0}\right),
\end{gathered}
$$

where $x$ is a point on the inner normal to $\Sigma$ at $x_{0}, x^{\prime}$ is its symmetric point with respect to $x_{0}$ and $\gamma\left(x_{0}\right)$ is given by $(2.2)$. 
If $\Sigma$ is a Lyapunov boundary, this result follows immediately from Theorem 1. This is not the case if we merely suppose $\Sigma \in C^{1}$.

Let $E$ be the operator

$$
E u=\sum_{|\alpha|=2 m} a_{\alpha} D^{\alpha} u
$$

where $a_{\alpha}$ are real constants. We suppose that the operator is elliptic, i.e.,

$$
Q(\xi)>0
$$

for any $\xi \in \mathbb{R}^{n} \backslash\{0\}$, where

$$
Q(\xi)=\sum_{|\alpha|=2 m} a_{\alpha} \xi^{\alpha}
$$

As it was shown by Fritz John [13, pp. 65-72] the functions

$$
S_{0}(x-y)=\frac{1}{4(2 \pi i)^{n-1}(2 m-1) !}\left(\Delta_{y}\right)^{(n-1) / 2} \int_{|\xi|=1} \frac{|(x-y) \cdot \xi|^{2 m-1}}{Q(\xi)} d \sigma_{\xi}
$$

for $n$ odd, and

$$
S_{0}(x-y)=\frac{-1}{(2 \pi i)^{n}(2 m) !}\left(\Delta_{y}\right)^{n / 2} \int_{|\xi|=1} \frac{|(x-y) \cdot \xi|^{2 m} \log |(x-y) \cdot \xi|}{Q(\xi)} d \sigma_{\xi}
$$

for $n$ even, provide a fundamental solution for (2.3).

We have the following theorem (see [4])

Theorem 3. Let $\Sigma \in C^{1}$. Let $\varphi \in L^{1}(\Sigma)$ and $x_{0} \in \Sigma$ be a Lebesgue point for $\varphi$. For any multi-index $\alpha$ with $|\alpha|=2 m-1$, we have

$$
\begin{gathered}
\lim _{x \rightarrow x_{0}}\left(\int_{\Sigma} \varphi(y) D_{y}^{\alpha}\left[S_{0}(x-y)\right] d \sigma_{y}-\int_{\Sigma} \varphi(y) D_{y}^{\alpha}\left[S_{0}\left(x^{\prime}-y\right)\right] d \sigma_{y}\right) \\
=-\frac{\nu^{\alpha}\left(x_{0}\right)}{Q\left(\nu\left(x_{0}\right)\right)} \varphi\left(x_{0}\right),
\end{gathered}
$$

where $x$ is a point on the inner normal to $\Sigma$ at $x_{0}$ and $x^{\prime}$ is its symmetric point with respect to $x_{0}$.

Proof. First write $\alpha=\alpha_{0}+\alpha_{1}$ with $\left|\alpha_{0}\right|=1,\left|\alpha_{1}\right|=2 m-2$ and then

$$
\begin{aligned}
& \int_{\Sigma} \varphi(y) D_{y}^{\alpha}\left[S_{0}(x-y)-S_{0}\left(x^{\prime}-y\right)\right] d \sigma_{y} \\
=- & D_{x}^{\alpha_{0}} \int_{\Sigma} \varphi(y) D_{y}^{\alpha_{1}}\left[S_{0}(x-y)-S_{0}\left(x^{\prime}-y\right)\right] d \sigma_{y} .
\end{aligned}
$$


Since $S_{0}(x)$ is essentially homogeneous of degree $2 m-n, D^{\alpha_{1}} S_{0}(x)$ is essentially homogeneous of degree $2-n$ and Theorem 2 gives

$$
\lim _{x \rightarrow x_{0}} D_{x}^{\alpha_{0}} \int_{\Sigma} \varphi(y) D_{y}^{\alpha_{1}}\left[S_{0}(x-y)-S_{0}\left(x^{\prime}-y\right)\right] d \sigma_{y}=2 \nu^{\alpha_{0}}\left(x_{0}\right) \gamma_{\alpha_{1}}\left(x_{0}\right) \varphi\left(x_{0}\right),
$$

where

$$
\gamma_{\alpha_{1}}\left(x_{0}\right)=-2 \pi^{2} \mathscr{F}\left(D^{\alpha_{1}} S_{0}\right)\left(\nu_{x_{0}}\right) .
$$

On the other hand, $E S_{0}=\delta$ and $\left(-4 \pi^{2}\right)^{m} Q(x) \mathscr{F}\left(S_{0}\right)(x)=1$. This leads to

$$
-2 \pi^{2} \mathscr{F}\left(D^{\alpha_{1}} S_{0}\right)(x)=-2 \pi^{2}(2 \pi i)^{2 m-2} x^{\alpha_{1}} \mathscr{F}\left(S_{0}\right)(x)=\frac{1}{2} \frac{x^{\alpha_{1}}}{Q(x)}
$$

and (2.6) follows from (2.7).

\section{The Principal Fundamental Solution in the Sense of Fichera}

In this section we are going to consider a more general operator (1.3) which we rewrite in the form

$$
E u=\sum_{|p|,|q|}^{0, m}(-1)^{|p|} a_{p q} D^{p} D^{q} u .
$$

As usual, we associate to (3.1) the bilinear form

$$
B(u, v)=\sum_{|p|,|q|}^{0, m} a_{p q} \int_{\Omega} D^{q} u D^{p} v d x .
$$

We suppose that $E$ is such that the Gårding inequality holds:

$$
B(u, u) \geqslant C\|u\|_{W^{m, 2}(\Omega)}^{2} \quad \forall u \in \stackrel{\circ}{C}^{\infty}(\Omega) .
$$

It is well known that condition (3.2) implies the ellipticity of the operator $E$. It is a more restrictive condition because the ellipticity of $E$ implies only a more general Gårding inequality

$$
B(u, u) \geqslant C\|u\|_{W^{m, 2}(\Omega)}^{2}-c\|u\|_{L^{2}(\Omega)}^{2} \quad \forall u \in \stackrel{\circ}{C}^{\infty}(\Omega)
$$

$(C, c>0)$.

Let us denote by $S_{0}$ the fundamental solution of the operator

$$
E^{(0)} u=\sum_{|\alpha|=2 m} a_{\alpha} D^{\alpha} u
$$

given by (2.4) or (2.5). The function $S_{0}(x-y)$ can be considered as a parametrix for the operator $E$. In particular, if

$$
u(x)=\int_{\mathbb{R}^{n}} \varphi(y) S_{0}(x-y) d y
$$


with $\varphi \in \stackrel{\circ}{C}^{\infty}\left(\mathbb{R}^{n}\right)$, then we have

$$
E u(x)=\varphi(x)+\int_{\mathbb{R}^{n}} \varphi(y) E_{x}^{(1)}\left[S_{0}(x-y)\right] d y,
$$

where

$$
E^{(1)} u=\sum_{|\alpha|<2 m} a_{\alpha} D^{\alpha} u
$$

Let $\mathscr{S}_{0}(x, y)=S_{0}(x-y)+g(x, y)$ be the Green function for the Dirichlet problem

$$
\begin{cases}E^{(0)} u=f & \text { in } T \\ D^{p} u=0 & \text { on } \partial T,|p| \leqslant m-1 .\end{cases}
$$

The existence of such a Green function is well known. Fichera himself gave a way for constructing it (see [9, Lecture 20]).

Let us consider the integral operator

$$
J \varphi(x)=\int_{T} \varphi(y) E_{x}\left[\mathscr{S}_{0}(x, y)\right] d y .
$$

Since $E_{x}\left[\mathscr{S}_{0}(x, y)\right]=E_{x}^{(1)}\left[\mathscr{S}_{0}(x, y)\right](x \neq y), J$ is a compact operator from $L^{2}(T)$ into itself.

Lemma 1. Let us suppose that the Gärding inequality (3.2) holds. The homogeneous equation

$$
\varphi+J \varphi=0
$$

where $J$ is given by (3.4), has no eigensolutions in $L^{2}(T)$.

Proof. Let $\varphi \in L^{2}(T)$ be a solution of (3.5). The potential

$$
u(x)=\int_{T} \varphi(y) \mathscr{S}_{0}(x, y) d y
$$

is a solution of the problem

$$
\begin{cases}E^{(0)} u=\varphi & \text { in } T, \\ D^{p} u=0 & \text { on } \partial T,|p| \leqslant m-1 .\end{cases}
$$

On the other hand, we have $E u=\varphi+J \varphi$ and $u$ is also a solution of the problem

$$
\begin{cases}E u=0 & \text { in } T \\ D^{p} u=0 & \text { on } \partial T,|p| \leqslant m-1 .\end{cases}
$$

From (3.2) it follows that this problem has no eigensolutions. Then $u=0$ and (3.6) shows that $\varphi=0$. 
We say that $F(x, y)$ is a fundamental solution in the sense of Fichera if it is a fundamental solution and, moreover,

$$
D_{x}^{p} F(x, y)=0, \quad x \in \partial T, \quad y \in T, \quad 0 \leqslant|p| \leqslant m-1,
$$

where $T$ is a ball containing $\Omega$ in its interior.

Theorem 4. Let $E$ be operator (1.3) and suppose that the Gärding inequality (3.2) holds. Let $T$ be an open ball containing $\bar{\Omega}$. There exists, in $T$, a principal fundamental solution in the sense of Fichera for the operator $E$.

Proof. Let $\mathscr{S}_{0}(x, y)$ be the Green function considered before. Since the Fredholm equation (3.5) has no eigensolutions, the equation

$$
\varphi+J \varphi=f
$$

has one and only one solution $\varphi \in L^{2}(T)$ given by

$$
\varphi(x)=f(x)+\int_{T} f(y) R(x, y) d y,
$$

where $R(x, y)$ is the resolvent kernel of the Fredholm equation (3.8). Define

$$
F(x, y)=\mathscr{S}_{0}(x, y)+\int_{T} \mathscr{S}_{0}(x, w) R(w, y) d w .
$$

$F(x, y)$ is the desired principal fundamental solution. Indeed, conditions (3.7) are obvious. Moreover, if

$$
u(x)=\int_{T} f(y) F(x, y) d y
$$

we can write

$$
u(x)=\int_{T} \varphi(y) \mathscr{S}_{0}(x, y) d y,
$$

where $\varphi$ is given by (3.9), and then

$$
E u=\varphi+J \varphi=f .
$$

If we consider the adjoint operator $E^{*}$ instead of $E$, then we obtain the principal fundamental solution $F^{*}(x, y)$ in the same way. By using standard arguments one can prove that $F^{*}(x, y)=F(y, x)$.

These are the main properties of $F$ and $F^{*}$ :

$$
\begin{aligned}
E_{x} F(x, y) & =0, \quad x \in T, \quad y \in T, \quad x \neq y ; \\
D_{x}^{p} F(x, y) & =0, \quad x \in \partial T, \quad y \in T, \quad 0 \leqslant|p| \leqslant m-1 ; \\
E_{y}^{*} F(x, y) & =0, \quad x \in T, \quad y \in T, \quad x \neq y ; \\
D_{y}^{p} F(x, y) & =0, \quad x \in T, \quad y \in \partial T, \quad 0 \leqslant|p| \leqslant m-1 ; \\
D_{x}^{p} D_{y}^{q} F(x, y) & =\mathcal{O}\left(D_{x}^{p} D_{y}^{q} S_{0}(x-y)\right), \quad 0 \leqslant|p|+|q|<2 m .
\end{aligned}
$$




\section{Completeness Theorems}

From now on we are going to suppose that the coefficient $a_{00}$ in (3.1) vanishes and that $\mathbb{R}^{n} \backslash \bar{\Omega}$ is connected.

We prove here our main result, which concerns the completeness of system (1.4). Here $\left\{\omega_{k}\right\}$ denotes a complete system of polynomial solutions of the equation $E \omega=0$. This means that any polynomial solution of this equation can be written as a finite linear combination of $\omega_{k}$.

It is well known that a complete system of harmonic polynomials can be obtained by ordering in one sequence the polynomials

$$
|x|^{h} Y_{h s}\left(\frac{x}{|x|}\right), \quad s=1, \ldots, p_{n h}, \quad h=0,1, \ldots,
$$

where $\left\{Y_{h s}\right\}\left(s=1, \ldots, p_{n h}, h=0,1, \ldots\right)$ is a complete system of ultra-spherical harmonics and $p_{n h}=(2 h+n-2)(h+n-3) ! /((n-2) ! h !)$.

It is possible to extend this classical procedure to the case of the polyharmonic operator $\Delta^{m}$. Indeed, a complete system of polyharmonic polynomials $\left\{\omega_{k}^{(m)}\right\}$ is given by

$$
\begin{gathered}
|x|^{h+2 j} Y_{h s}\left(\frac{x}{|x|}\right), \quad j=0, \ldots, m-1, \quad s=1, \ldots, p_{n h}, \quad h=0,1, \ldots, \\
\left.\left(p_{n h}=(2 h+n-2)(h+n-3) ! /((n-2) ! h !)\right) \text { (see, e.g., }[5]\right) .
\end{gathered}
$$

In the particular case of two independent variables, a system of polynomial solutions of the equation $E u=0$ was constructed in [1] in the following way. The operator $E$ can be written as $E=E_{1}^{k_{1}} \ldots E_{m}^{k_{m}}$, where

$$
E_{i}=a_{0}^{(i)} \frac{\partial^{2}}{\partial x^{2}}+a_{1}^{(i)} \frac{\partial^{2}}{\partial x \partial y}+a_{2}^{(i)} \frac{\partial^{2}}{\partial y^{2}}
$$

are elliptic operators, and

$$
Q_{i}(w)=a_{0}^{(i)} w^{2}+a_{1}^{(i)} w+a_{2}^{(i)} .
$$

Let $\lambda_{i} \in \mathbb{C}$ such that $Q_{i}\left(\lambda_{i}\right)=Q_{i}\left(\bar{\lambda}_{i}\right)=0$ with $\mathscr{I} m \lambda_{i}<0$ and $\lambda_{i} \neq \lambda_{j}$ if $i \neq j$. If $p$ is a homogenous polynomial of degree $k \geqslant 2 m$, we have that $L p=0$ if and only if $p$ is a finite linear combination of the following polynomials

$$
\begin{cases}\varrho_{1}^{h}\left(\lambda_{1} x+y\right)^{k-2 h} ; \varrho_{1}^{h}\left(\bar{\lambda}_{1} x+y\right)^{k-2 h}, & h=0,1, \ldots, k_{1}-1, \\ \ldots & \\ \varrho_{m}^{h}\left(\lambda_{m} x+y\right)^{k-2 h} ; \varrho_{m}^{h}\left(\bar{\lambda}_{m} x+y\right)^{k-2 h}, \quad h=0,1, \ldots, k_{m}-1\end{cases}
$$

where $\varrho_{i}=a_{2}^{(i)} x^{2}-a_{1}^{(i)} x y+a_{0}^{(i)} y^{2}$. These polynomials are complex. A complete system of real polynomial solutions is given by

$$
\begin{cases}\varrho_{1}^{h} \mathscr{R} e\left(\lambda_{1} x+y\right)^{k-2 h} ; \varrho_{1}^{h} \mathscr{I} m\left(\lambda_{1} x+y\right)^{k-2 h}, & h=0,1, \ldots, k_{1}-1 \\ \ldots & \\ \varrho_{m}^{h} \mathscr{R} e\left(\lambda_{m} x+y\right)^{k-2 h} ; \varrho_{m}^{h} \mathscr{I} m\left(\lambda_{m} x+y\right)^{k-2 h}, & h=0,1, \ldots, k_{m}-1 .\end{cases}
$$


For the construction of a system of polynomial solutions for more general operators, we refer to $[19,21,22]$.

Before proving our main result we need a completeness result for a particular class of potentials. Let $T$ be a ball containing $\bar{\Omega}$ and denote by $F(x, y)$ the principal fundamental solution given by Theorem 4 . Let $\Omega_{1}$ be a bounded domain such that $\bar{\Omega} \subset \Omega_{1}, \bar{\Omega}_{1} \subset T$ and $\Sigma_{1}=\partial \Omega_{1} \in C^{1}$. Denote by $\mathscr{S}\left(\Omega_{1}\right)$ the class of potentials

$$
u(x)=\sum_{|\alpha| \leqslant m-1} \int_{\Sigma_{1}} \varphi_{\alpha}(y) D_{y}^{\alpha}[F(x, y)] d \sigma_{y}
$$

with $\varphi_{\alpha}$ varying in $C^{0}\left(\Sigma_{1}\right)$. If $u \in \mathscr{S}\left(\Omega_{1}\right)$, then $u \in C^{\infty}\left(\Omega_{1}\right) \cap C^{m-1}\left(\bar{\Omega}_{1}\right)$ and $E u=0$ in $\Omega_{1}$.

Theorem 5. Let E be operator (3.1) and suppose that the Garding inequality (3.2) holds. Then the system

$$
\left\{\left(u, \partial_{\nu} u, \ldots, \partial_{\nu}^{m-1} u\right) \mid u \in \mathscr{S}\left(\Omega_{1}\right)\right\}
$$

is complete in $\left[L^{p}(\Sigma)\right]^{m}(1 \leqslant p<\infty)$.

Proof. We have to show that, if $\left(\beta_{1}, \ldots, \beta_{m}\right) \in\left[L^{q}(\Sigma)\right]^{m}(q=p /(p-1)$ if $p>1$, $q=\infty$ if $p=1$ ) is such that

$$
\sum_{h=1}^{m} \int_{\Sigma} \beta_{h} \partial_{\nu}^{h-1} u d \sigma=0 \quad \forall u \in \mathscr{S}\left(\Omega_{1}\right),
$$

then $\beta_{1}=\cdots=\beta_{m}=0$.

Since $u \in \mathscr{S}\left(\Omega_{1}\right)$ is given by (4.1), from (4.2) we find

$$
\sum_{|\alpha| \leqslant m-1} \sum_{h=1}^{m} \int_{\Sigma} \beta_{h}(x) \partial_{\nu_{x}}^{h-1}\left(\int_{\Sigma_{1}} \varphi_{\alpha}(y) D_{y}^{\alpha}[F(x, y)] d \sigma_{y}\right) d \sigma_{x}=0,
$$

i.e.,

$$
\sum_{|\alpha| \leqslant m-1} \sum_{h=1}^{m} \int_{\Sigma_{1}} \varphi_{\alpha}(y) D_{y}^{\alpha}\left(\int_{\Sigma} \beta_{h}(x) \partial_{\nu_{x}}^{h-1}[F(x, y)] d \sigma_{x}\right) d \sigma_{y}=0 .
$$

The arbitrariness of $\varphi_{\alpha}$ leads to

$$
D^{\alpha} \Gamma(y)=0 \quad \forall y \in \Sigma_{1}, \quad|\alpha| \leqslant m-1,
$$

where

$$
\Gamma(y)=\sum_{h=1}^{m} \int_{\Sigma} \beta_{h}(x) \partial_{\nu_{x}}^{h-1}[F(x, y)] d \sigma_{x} .
$$

The function $\Gamma$ is a solution of the following problem

$$
\begin{cases}E^{*} \Gamma=0 & \text { in } T \backslash \bar{\Omega}_{1}, \\ D^{p} \Gamma=0 & \text { on } \partial T \cup \partial \Omega_{1},|p| \leqslant m-1,\end{cases}
$$


and then $\Gamma(y)=0$ in $T \backslash \bar{\Omega}_{1}$. On the other hand, $\Gamma$ is a solution of the equation $E^{*} \Gamma=0$ in $T \backslash \bar{\Omega}$. Then $\Gamma$ is analytic in $T \backslash \bar{\Omega}$ and since $\Gamma$ vanishes in $T \backslash \Omega_{1}$, we have $\Gamma=0$ in $T \backslash \bar{\Omega}$.

This implies that $\Gamma$ is a solution of the problem

$$
\begin{cases}E^{*} \Gamma=0 & \text { in } \Omega, \\ D^{p} \Gamma=0 & \text { on } \partial \Omega,|p| \leqslant m-1\end{cases}
$$

and then

$$
\Gamma(x)=0 \quad \forall x \in \mathbb{R}^{n} \backslash \Sigma .
$$

Let now $\alpha$ be a multi-index with $|\alpha|=m$; we have

$$
D^{\alpha} \Gamma(x)=0 \quad \forall x \in \mathbb{R}^{n} \backslash \Sigma,
$$

i.e.,

$$
\sum_{h=1}^{m} \int_{\Sigma} \beta_{h}(y) \partial_{\nu_{y}}^{h-1} D_{x}^{\alpha}[F(y, x)] d \sigma_{y}=0 \quad \forall y \in \mathbb{R}^{n} \backslash \Sigma .
$$

This implies

$$
\lim _{x \rightarrow x_{0}} \sum_{h=1}^{m} \int_{\Sigma} \beta_{h}(y) \partial_{\nu_{y}}^{h-1} D_{x}^{\alpha}\left[F(y, x)-F\left(y, x^{\prime}\right)\right] d \sigma_{y}=0
$$

for any $x_{0} \in \Sigma$, where $x, x^{\prime}$ have the same meaning as in Theorem 3 .

On the other hand, due to the weak singularities of the kernels, we have

$$
\lim _{x \rightarrow x_{0}} \sum_{h=1}^{m-1} \int_{\Sigma} \beta_{h}(y) \partial_{\nu_{y}}^{h-1} D_{x}^{\alpha}\left[F(y, x)-F\left(y, x^{\prime}\right)\right] d \sigma_{y}=0
$$

and then

$$
\lim _{x \rightarrow x_{0}} \int_{\Sigma} \beta_{m}(y) \partial_{\nu_{y}}^{m-1} D_{x}^{\alpha}\left[F(y, x)-F\left(y, x^{\prime}\right)\right] d \sigma_{y}=0 .
$$

In view of (2.6) and (3.10), we have also

$$
\begin{aligned}
& \lim _{x \rightarrow x_{0}} \int_{\Sigma} \beta_{m}(y) \partial_{\nu_{y}}^{m-1} D_{x}^{\alpha}\left[F(y, x)-F\left(y, x^{\prime}\right)\right] d \sigma_{y} \\
& \quad=\lim _{x \rightarrow x_{0}} \sum_{|\beta|=m-1} \frac{(m-1) !}{\beta !} \int_{\Sigma} \beta_{m}(y) \nu^{\beta}(y) D_{x}^{\alpha} D_{y}^{\beta}\left[F(y, x)-F\left(y, x^{\prime}\right)\right] d \sigma_{y} \\
& =\lim _{x \rightarrow x_{0}} \sum_{|\beta|=m-1} \frac{(m-1) !}{\beta !} \int_{\Sigma} \beta_{m}(y) \nu^{\beta}(y) D_{x}^{\alpha} D_{y}^{\beta}\left[\mathscr{S}_{0}(y, x)-\mathscr{S}_{0}\left(y, x^{\prime}\right)\right] d \sigma_{y} \\
& =(-1)^{m} \lim _{x \rightarrow x_{0}} \sum_{|\beta|=m-1} \frac{(m-1) !}{\beta !} \int_{\Sigma} \beta_{m}(y) \nu^{\beta}(y) D_{y}^{\alpha+\beta}\left[S_{0}(x-y)-S_{0}\left(x^{\prime}-y\right)\right] d \sigma_{y}
\end{aligned}
$$




$$
\begin{array}{r}
=(-1)^{m-1} \frac{\nu^{\alpha}\left(x_{0}\right)}{Q\left(\nu\left(x_{0}\right)\right)}\left(\sum_{|\beta|=m-1} \frac{(m-1) !}{\beta !}\left(\nu^{\beta}\left(x_{0}\right)\right)^{2}\right) \beta_{m}\left(x_{0}\right) \\
=(-1)^{m-1} \frac{\nu^{\alpha}\left(x_{0}\right)}{Q\left(\nu\left(x_{0}\right)\right)} \beta_{m}\left(x_{0}\right)
\end{array}
$$

for almost every $x_{0} \in \Sigma$.

This leads to

$$
\nu^{\alpha}\left(x_{0}\right) \beta_{m}\left(x_{0}\right)=0
$$

almost everywhere on $\Sigma$ and for any multi-index $\alpha$ with $|\alpha|=m$. Then we have also

$$
\beta_{m}\left(x_{0}\right)=\sum_{|\alpha|=m} \frac{m !}{\alpha !}\left(\nu^{\alpha}\left(x_{0}\right)\right)^{2} \beta_{m}\left(x_{0}\right)=0
$$

i.e., $\beta_{m}=0$ almost everywhere on $\Sigma$.

Now (4.3) implies

$$
\lim _{x \rightarrow x_{0}} \sum_{h=1}^{m-1} \int_{\Sigma} \beta_{h}(y) \partial_{\nu_{y}}^{h-1} D_{x}^{\alpha}\left[F(y, x)-F\left(y, x^{\prime}\right)\right] d \sigma_{y}=0
$$

for any multi-index $\alpha$ with $|\alpha|=m+1$. An argument similar to the previous one leads to $\beta_{m-1}=0$ a.e. and the result follows by induction.

The next Theorem is due to Malgrange [16]:

Theorem 6. Let $\Omega_{1}$ be a convex domain. Every solution of the equation $E u=0$ is the limit, in $C^{\infty}\left(\Omega_{1}\right)$, of polynomial solutions of the same equation if and only if all the irreducible factors (over $\mathbb{C}$ ) of the polynomial $Q(\xi)$ vanish at $\xi=0$.

We briefly say that the polynomial $Q$ satisfies the $M$-condition if all its irreducible factors over $\mathbb{C}$ vanish at the origin.

Theorem 7. Let E be operator (3.1) and suppose that the Garding inequality (3.2) holds. Let us denote by $Q$ the characteristic polynomial

$$
Q(\xi)=\sum_{|p|,|q|}^{0, m}(-1)^{|p|} a_{p q} \xi^{p+q}
$$

Let $\left\{\omega_{k}\right\}$ be a complete system of polynomial solutions of the equation Eu= 0 . The system $\left\{\left(\omega_{k}, \partial_{\nu} \omega_{k}, \ldots, \partial_{\nu}^{m-1} \omega_{k}\right)\right\}$ is complete in $\left[L^{p}(\Sigma)\right]^{m}$ if and only if polynomial (4.4) satisfies the $M$-condition.

Proof. Sufficiency. Let us suppose that the $M$-condition is satisfied.

Fix a convex domain $\Omega_{1}$ with a $C^{1}$ boundary such that $\bar{\Omega} \subset \Omega_{1}$. In view of Theorem 5 , given $\left(f_{1}, \ldots, f_{m}\right) \in\left[L^{p}(\Sigma)\right]^{m}$ and $\varepsilon>0$, there exists $u \in \mathscr{S}\left(\Omega_{1}\right)$ such that

$$
\sum_{h=1}^{m}\left\|f_{h}-\partial_{\nu}^{h-1} u\right\|_{L^{p}(\Sigma)}<\varepsilon .
$$


Theorem 6 shows that we can find a sequence of polynomial solutions of the equations $E p=0$ converging to $u$ in $C^{\infty}\left(\Omega_{1}\right)$. This implies that we can find a polynomial solution $\omega$ of the equation $E \omega=0$ such that

$$
\sum_{h=1}^{m}\left\|\partial_{\nu}^{h-1} u-\partial_{\nu}^{h-1} \omega\right\|_{L^{p}(\Sigma)}<\varepsilon .
$$

We have shown that for any $\varepsilon>0$ there exists a polynomial solution $\omega$ of the equation $E \omega=0$ such that

$$
\sum_{h=1}^{m}\left\|f_{h}-\partial_{\nu}^{h-1} \omega\right\|_{L^{p}(\Sigma)}<2 \varepsilon
$$

and the sufficiency is proved.

Necessity. Fix an open ball $T$ such that $\bar{T} \subset \Omega$. Consider $f \in \mathcal{U}(T)$, where

$$
\mathcal{U}(T)=\left\{f \in C^{\infty}(T) \mid E f=0\right\} .
$$

Let $K$ be a compact set contained in $T$. Let $\widetilde{T}$ be a concentric ball such that $K \subset \widetilde{T} \subset T$.

In view of Theorem 5 , for any $\varepsilon>0$ we can find $v \in \mathscr{S}(\Omega)$ such that

$$
\sum_{h=1}^{m}\left\|\partial_{\nu}^{h-1} f-\partial_{\nu}^{h-1} v\right\|_{L^{p}(\partial \widetilde{T})}<\varepsilon .
$$

Note that $v \in C^{\infty}(\Omega) \cap C^{m-1}(\bar{\Omega})$. By hypothesis, there exists a polynomial solution $\omega$ such that

$$
\sum_{h=1}^{m}\left\|\partial_{\nu}^{h-1} v-\partial_{\nu}^{h-1} \omega\right\|_{L^{p}(\Sigma)}<\varepsilon .
$$

By standard arguments, for any multi-index $\alpha$ there exists a constant $C$ (which depends on $E, \alpha, K, \Omega$ and $p$ ) such that

$$
\max _{x \in K}\left|D^{\alpha} v(x)-D^{\alpha} \omega(x)\right| \leqslant C \sum_{h=1}^{m}\left\|\partial_{\nu}^{h-1} v-\partial_{\nu}^{h-1} \omega\right\|_{L^{p}(\Sigma)} .
$$

In the same way there exists $\widetilde{C}$ such that

$$
\max _{x \in K}\left|D^{\alpha} f(x)-D^{\alpha} v(x)\right| \leqslant \widetilde{C} \sum_{h=1}^{m}\left\|\partial_{\nu}^{h-1} f-\partial_{\nu}^{h-1} v\right\|_{L^{p}(\partial \widetilde{T})} .
$$

This shows that there exists a sequence $\left\{\omega^{(n)}\right\}$ of polynomial solutions such that, for any multi-index $\alpha$, the sequence $D^{\alpha} \omega^{(n)}$ uniformly converges to $D^{\alpha} f$ on $K$.

Let $\mu \in \mathscr{E}^{\prime}(T)$ be a distribution with compact support such that

$$
\left\langle\mu, \omega_{k}\right\rangle=0, \quad k=1,2, \ldots .
$$

Let $U \subset T$ be a bounded domain containing the support of $\mu$. From what we have just said, given $f \in \mathcal{U}(T)$, there exists a sequence $\left\{\omega^{(n)}\right\}$ of polynomial solutions of the equation $E \omega=0$ in $\Omega$ such that, for any multi-index $\alpha, D^{\alpha} \omega^{(n)}$ 
uniformly converges to $D^{\alpha} f$ on $\bar{U}$. Moreover, for some constants $C$ and $s$ the inequality

$$
|\langle\mu, \varphi\rangle| \leqslant C \sum_{|\alpha| \leqslant s} \sup _{x \in U}\left|D^{\alpha} \varphi(x)\right|
$$

is valid for any $\varphi \in C^{\infty}(T)$ (see, e.g., [12, p. 44]). Keeping in mind (4.5), we find

$$
\langle\mu, f\rangle=\lim _{n \rightarrow \infty}\left\langle\mu, \omega^{(n)}\right\rangle=0 .
$$

This shows that the system $\left\{\omega_{k}\right\}$ is complete in the class $\mathcal{U}(T)$ in the usual topology of $\mathscr{E}(T) \equiv C^{\infty}(T)$. By Malgrange's result (Theorem 6) the $M$ condition is satisfied and the proof is complete.

Remark. If the operator $E$ does not contain lower order terms and is elliptic, the Gårding inequality (3.2) and the $M$-condition are both satisfied. Therefore the completeness results proved in [4] are contained in Theorem 7.

Remark. The $M$-condition and the Gårding inequality (3.2) are independent of each other. For example, the operator $-\Delta u+u$ satisfies $(3.2)$, but $Q(\xi)=$ $|\xi|^{2}+1$ does not satisfy the $M$-condition.

In order to construct an example of an elliptic operator which satisfies the $M$-condition but not inequality (3.2), consider $u_{0} \in \stackrel{\circ}{ }^{\infty}(\Omega), \Omega \subset \mathbb{R}^{2}$ and choose $\lambda$ such that

$$
\lambda>\left(\int_{\Omega}\left(\frac{\partial u_{0}}{\partial x}\right)^{2} d x d y\right)^{-1} \int_{\Omega}\left[\left(\frac{\partial^{2} u_{0}}{\partial x^{2}}\right)^{2}+\left(\frac{\partial^{2} u_{0}}{\partial y^{2}}\right)^{2}\right] d x d y .
$$

The elliptic operator

$$
E u=\frac{\partial^{4} u}{\partial x^{4}}+\frac{\partial^{4} u}{\partial y^{4}}+\lambda \frac{\partial^{2} u}{\partial x^{2}}
$$

satisfies (3.3), but it does not satisfy (3.2). Indeed, the inequality

$$
\int_{\Omega}\left[\left(\frac{\partial^{2} u}{\partial x^{2}}\right)^{2}+\left(\frac{\partial^{2} u}{\partial y^{2}}\right)^{2}\right] d x d y-\lambda \int_{\Omega}\left(\frac{\partial u}{\partial x}\right)^{2} d x d y \geqslant C\|u\|_{W^{2,2}(\Omega)}^{2}
$$

cannot hold for any $u \in \stackrel{\circ}{C}^{\infty}(\Omega)$ because of (4.6).

The characteristic polynomial of (4.7) is

$$
Q(x, y)=x^{4}+y^{4}+\lambda x^{2}
$$

it satisfies the $M$-condition because it is irreducible over $\mathbb{C}$.

\section{REFERENCES}

1. A. Cialdea, Teoremi di completezza connessi con equazioni ellittiche di ordine superiore in due variabili in un campo con contorno angoloso, Rend. Circ. Mat. Palermo (2) 34(1985), 32-49. 
2. A. Cialdea, The multiple layer potential for the biharmonic equation in $n$ variables. Atti Accad. Naz. Lincei Cl. Sci. Fis. Mat. Natur. Rend. Lincei (9) Mat. Appl. 3(1992), No. 4, 241-259.

3. A. Cialdea, A general theory of hypersurface potentials. Ann. Mat. Pura Appl. (4) 168(1995), 37-61

4. A. Cialdea, Completeness Theorems: Fichera's fundamental results and some new contributions (to appear).

5. A. Cialdea and A. Malaspina, Completeness Theorems for the Dirichlet Problem for the Polyharmonic Equation, Rend. Accad. Naz. Sci. XL (to appear).

6. L. Ehrenpreis, Solution of some problems of division. I. Division by a polynomial of derivation. Amer. J. Math. 76(1954), 883-903.

7. G. Fichera, Teoremi di completezza sulla frontiera di un dominio per taluni sistemi di funzioni. (Italian) Ann. Mat. Pura Appl. (4) 27(1948), 1-28.

8. G. FicherA, Linear elliptic equations of higher order in two independent variables and singular integral equations, with applications to anistropic inhomogeneous elasticity. Partical differential equations and continuum mechanics, 55-80. Univ. of Wisconsin Press, Madison, Wis., 1961.

9. G. FicherA, Linear elliptic differential systems and eigenvalue problems. Lecture Notes in Mathematics, 8. Springer-Verlag, Berlin-New York, 1965.

10. G. Fichera, The problem of the completeness of systems of particular solutions of partial differential equations. Numerical mathematics (Sympos., Inst. Appl. Math., Univ. Hamburg, Hamburg, 1979), 25-41, Internat. Ser. Numer. Math., 49, Birkhäuser, BaselBoston, Mass., 1979.

11. L. Hörmander, On the theory of general partial differential operators. Acta Math. 94(1955), 161-248.

12. L. HöRMANDER, The analysis of linear partial differential operators. I. Distribution theory and Fourier analysis. Grundlehren der Mathematischen Wissenschaften [Fundamental Principles of Mathematical Sciences], 256. Springer-Verlag, Berlin, 1983.

13. F. John, Plane waves and spherical means applied to partial differential equations. Interscience Publishers, New York-London, 1955.

14. H. KÖNIG, An explicit formula for fundamental solutions of linear partial differential equations with constant coefficients. Proc. Amer. Math. Soc. 120(1994), No. 4, 13151318.

15. V. D. Kupradze, T. G. Gegelia, M. O. Basheleishryili, and T. V. BurchuLADZE, Three-dimensional problems of the mathematical theory of elasticity and thermoelasticity. (Translated from the Russian) North-Holland Series in Applied Mathematics and Mechanics, 25. North-Holland Publishing Co., Amsterdam-New York, 1979; Russian Original: Nauka, Moscow, 1976.

16. B. Malgrange, Existence et approximation des solutions des équations aux dérivées partielles et des équations de convolution. Ann. Inst. Fourier, Grenoble 6(1955-1956), $271-355$.

17. N. Ortner and P. Wagner, A short proof of the Malgrange-Ehrenpreis theorem. Functional analysis. Proceedings of the First International Workshop held at Trier University, Trier, September 26 - October 1, 1994, 343-352, de Gruyter, Berlin, 1996.

18. N. Ortner and P. Wagner, A survey on explicit representation formulae for fundamental solutions of linear partial differential operators. Acta Appl. Math. 47(1997), No. 1, 101-124.

19. P. S. Pedersen, A basis for polynomial solutions to systems of linear constant coefficient PDE's. Adv. Math. 117(1996), No. 1, 157-163. 
20. M. G. Platone Garroni, Matrici fondamentali principali per sistemi fortemente ellittici. (Italian) Atti del Convegno sulle Equazioni alle Derivate Parziali (Bologna, 1967), 72-88. Edizioni "Oderisi", Gubbio, 1967.

21. B. REZnick, Homogeneous polynomial solutions to constant coefficient PDE's. Adv. Math. 117(1996), No. 2, 179-192.

22. S. P. Sмiтh, Polynomial solutions to constant coefficient differential equations. Trans. Amer. Math. Soc. 329(1992), No. 2, 551-569.

23. F. TRÈvEs, Linear partial differential equations with constant coefficients: Existence, approximation and regularity of solutions. Mathematics and its Applications, Vol. 6. Gordon and Breach Science Publishers, New York-London-Paris, 1966.

24. P. WAgner, On the explicit calculation of fundamental solutions. Special issue dedicated to John Horváth. J. Math. Anal. Appl. 297(2004), No. 2, 404-418.

(Received 30.07.2006)

Author's address:

Dipartimento di Matematica

Università della Basilicata

Viale dell'Ateneo Lucano 10, 85100, Potenza

Italy

E-mail: cialdea@email.it 